Paget ${ }^{3}$ observed, "there will be danger from the most gentle catheterism." I have seen low densities in urine rise steadily to normal and remain so after the tension has been taken off micturition by the induction of atrophy of the prostate. It is not unlikely that we may find in vasectomy another means for removing intra-renal tension. It must be remembered that it sometimes takes but very little to render a prostate obstructive. Sir Henry Thompson pointed this out in connexion with his important studies on the pathology of this gland. I have seen enough to convince me that a small amount of shrinkage, provided it takes place in or indirectly affects the right spot, makes all the difference as to whether a man leads a life of comfort or not.

It is not unlikely that this operation will be extended to other disorders of the urinary organs. Though the normal function of these ducts is that of conveying the seminal fluid from the testes to the vesicles and prostatic urethra they are equally capable of transmitting micro-organisms in both upward and downward directions. In this sense they may therefore be regarded as distributors of disease. Some years ago, soon after I had published a short article ${ }^{4}$ on division of the vas deferens relative to prostatic hypertrophy, I saw a delicate-looking young man with a strong tuberculous family history with a nodule in his left testicle remaining after an acute gonorrbcea. This nodule was deemed to be tuberculous or likely to become so. The question then raised had reference more especially to the removal of this by operation on the ground of its suspicious nature. The urine was healthy and so was the opposite testicle and its tubes. Nor was I able to discover any evidence of deposit so far as the finger could reach either in the vas or the prostate. The patient, who had had some medical education, was anxious that either the nodule or the testis should be removed. I did not feel disposed to recommend either course. Having regard to the fact that the disease appeared limited to the nodule and that any infection would probably pass along the canal of the vas deferens I proposed to excise a portion of the latter. This was done and the wound healed in a few days. A year afterwards it was found that the testis and nodule had both completely atrophied and no signs of tuberculous infection conld be detected. The patient's health and sexual powers remain unimpaired.

The second group of cases of transference of infection through these ducts may be illustrated by the inflammations that take place of the testes and tubes occurring in connexion with some cases of prostatic hypertrophy where catheterism is necessary and often difficult. This is a complication which when frequently repeated seriously adds to the gravity and pain of these disorders. Farly in the year 1896 I saw a man approaching seventy years of age, otherwise in good health, who, in addition to much prostatitis, repeatedly suffered from most painful epididymitis in one or both organs, though sexual power had ceased for some years. He was dependent on the catheter and these attacks had been going on for some months, almost entirely confining him to bed and preventing him attending to his business. I divided his vasa for him with great relief so far as his prostatic symptoms generally were concerned and since this was done he has had no further trouble with his testicles.

I have tried various methods for resecting the vasa. The simplest appears to consist in rendering the vas superficial by manipulation of the scretum and making a small incision over it. It can then be easily seized with a Spencer Wells' clamp forceps and brought to the surface, where it is precisely isolated by a little scraping and a blunt hook or aneurysm needle passed beneath it. A loop is included by a silk ligature and the free portion removed by scissors. To ligature the duct is insufficient, it being necessary to resect a portion of it. After the loop has thus been removed the stump is returned with the ligature cut short and the little wound in then closed with a suture or collodion. Union usually takes place in the course of a day or two. In this way the operation can be performed quickly and almost bloodlessly.

In concluding these remarks I need hardly suggest that patients should invariably be made to understand, whatever their ages may be, that though division of one duct does not interfere with the function of generation the subsequent section of the second duct entirely and permanently extinguishes any sexual power that the individual may bave previously possessed. The operation may therefore be said to be restricted to that period of life and to coexisting circumstances when the genital function relative to the urinary disability is ceasing or has ceased to be a matter for consideration. The cases must be extremely rare where in earlier years the damage occasioned by the hopeless loss of all control over this function calls for such measures as vasectomy would undoubtedly supply. Further, I would repeat in connexion with the latter operation as well as with other procedures of a like nature that they are only applicable to grave varieties of prostatic disease and other complications arising out of them. When we recognise how many elderly men carry on long and useful lives who are more or less dependent upon the aid their catheter affords it is hardly necessary to say that such measures can only apply to the exceptions and not to the rules. The latter are already adequately provided for, whilst in the interests of the former all proved methods either of cure or of relief must receive our careful and unbiased consideration.

Lower Berkeley-street, $W$.

\section{A NOTE ON CERTAIN POINTS OF TECHNIQUE.}

BY A. E. WRIGHT, M.D. DUB.,

PROFESSOR OF PATHOLOGY, ARMY MEDICAL SCHOOL, NETLEY.

\section{(1) ON a Rapid AND Conventent Method of} Sterilisting Syringes and SURGICAL Instruments.

THE ordinary methods of sterilising syringes or instruments by either prolonged boiling in water, or prolonged soakage in antiseptic solutions, are unsatisfactory in several respects. In the first place both these methods of sterilisation involve a considerable loss of time; and, in the second place, the repeated application of these methods is very destructive both to surgical instruments and also to the asbestos plungers which are employed in syringes. These defects, which are inseparable from the ordinary methods of sterilisation, can be very simply avoided by employing as our sterilising agent olive oil at from $160^{\circ}$ to $180^{\circ} \mathrm{C}$. instead of water at $100^{\circ} \mathrm{C}$. In order to obtain complete sterilisation by this method it suffices in the case of a surgical instrument to dip it for an instant into the hot oil. In the case of a syringe it suffices to fill in the syringe twice with oil at the stated temperature. The temperature may be determined either by a thermometer, or if a thermometer is not at hand by a piece of ordinary breadcrumb. It will be found that the bread-crumb will become brown and crisp as soon as a temperature of from $160^{\circ}$ to $180^{\circ} \mathrm{C}$. is reached. This method of sterilisation has been found very convenient in the ordinary routine work of the laboratory. It will probably also prove of service in surgical practice. It is especially applicable in cases where there is no time for the prolonged soakage of instruments and further in cases where an instrument has become accidentally contaminated in the course of an operation. The method of employing hot oil as a sterilising agent is also a method which would appear to be readily applicable in ordinary practice for the sterilisation of syringes. The olive oil may be heated in a spoon over a spirit lamp, and the heat may be tested by the proposed bread-crumb thermometer. It is to be noted that the method is not applicable to the sterilisation of indiarubber tubing.

The above method of sterilisation has been tested by dipping instruments into, and filling in syringes with, putrescent fluids from manure heaps and cultures which contained very resistant spores. The test-cultivations which were made from these instruments and syringes after the sterilising operations which have been described remained in all cases absolutely sterile. An incidental advantage of the method of sterilising syringes by hot oil is that if the needles are sterilised in the hot oil before they are replaced in the case they are thereby effectually protected against rust.

(2) Method of Emptying Air out of a Syringe Without Contaminating THe OUTSIDE OF THE Syringe with the CoNTents.

A convenient way of effecting this object is the following. A small bottle of any strong antiseptic solution is coversd over with one of the rubber caps which are fitted on to testtubes to prevent the drying nn of kacterial cul: ure media. 
This bottle is held cap downwards and the needle of the eyringe which is to be emptied of air is thrust through into tbe antiseptic solution. The piston of the syringe may then be pushed home until all the bubbles have been expelled without any fear of contaminating the outside of the needle or syringe. It is unnecessary to sterilise the outside of the rubber cap before puncturing it with the hypodermic needle, for the needle will be again quite sterile when it comes ont from the antiseptic.

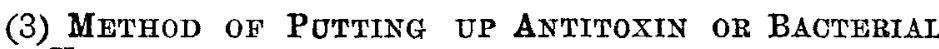
VACCINES IN SUCH A WAY THAT THEY MAY BE DIRECTLY DRAWN OFF IN AN ASEPTIC MANNER into A HYPODERMIC SrRINGT.

The method in which antitoxin is usually put up appears to be faulty in two respects. First, it is somewhat difficult to sterilise the chink between the rubber bung and the neck of the bottle. Secondly, inasmuch as it is impossible to get the needle of the syringe down to the bottom of the narrow mouthed bottles which are employed it becomes necessary to pour out the fluid into some shallower vessel. This involves as an incidental inconvenience the sterilisation of the vessel which is employed for receiving the antitoxin.

These inconveniences are perhaps most simply avoided by putting up the antitoxin in bottles which are fitted with stout close-fitting rubber caps in lieu of the usual rubber bungs. After the rubber caps have been placed in position they are to be rendered perfectly air-tight by dipping the neck of the bottles into a vessel containing sterilised and melted paraffin The contents of the bottle can then be drawn off as follows. First, the outside of the cap is re-sterilised by dipping it either into a hot antiseptic solution or into the hot oil which has served for the sterilisation of the syringe. Holding the bottle still in the inverted position the rubber cap is now to be pierced with the sterilised needle of the hypodermic syringe. The puncture which is thus produced serves only to admit air to the bottle. The needle is therefore now withdrawn and it is re-introduced into the antitoxin by puncturing the cap a second time. The contents of the bottle are now simply drawn off into the syringe.

This method of putting up antitoxin bas now been tested in practice for some considerable time. It has answered satisfactorily even in the case of the antitoxin which has been sent abroad. The method has also been employed with success for putting up the bacterial vaccine, which has been used in connexion with several considerable series of typhoid vaccinations. In these cases the bottle of bacterial vaccine was fixed in an inverted position in a clamp and the syringe was refilled time after time by simply puncturing through the indiarubber cap. When a sufficiency of vaccine had been drawn off the outside of the cap was re-sterilised and was then recoated with paraffin.

(4) Method of Preparing Aseptio Physiological STYPTICS.

The proposal which I put forward several years ago to utilise physiological and painless, in lieu of escharotic and painful, styptics in the arrest of hæmorrbage ${ }^{1}$ has not yet been practically exploited. None the less physiological styptics are calculated to be of eminent utility not only on the battle-field, but also in the minor surgery of the household, and above all, in the treatment of severe epistaxis and bromophilic bleeding. In these last cases, at any rate, the mediæval methods of arresting hæmorrhage by means of escharotic styptics would appear to be now no longer admissible.

So far as this neglect to take advantage of the proposed physiological styptics is not imputable to a lack of enterprise on the part of the manufacturing chemist, it has been, no doubt, due, first, to the fact that the method of preparaif I which was proposed was not a method that offered any absolnte guarantee for the aseptic condition of the styptic; and, secondly, to the fact that no convenient method of testing these styptics was proposed.

The following method of preparing a physiological styptic would appear to offer the required guarantee for the asepticity of the preparation. Fresh and finely.minced thymus gland is extracted for from twelve to twenty-four hours with from ten to twenty times its weight of a solution containing 1 per cent. of carbolic acid and 1 per cent. of common salt. An addition of 0.5 per cent. of calcium chloride cryst. is to be made to this fluid after it has been strained through a piece

1 The LaNCET, Feb. 25tb, 1893, p 435, and Brit. Med, Jour., 1891. of sterile muslin. Lastly the styptic solution is rendered very faintly alkaline. It is to be shaken up before use and is to be mixed with the effused blood in the proportion of about one part of styptic to ten of blood.

A less potent but an always accessible form of styptic may be very simply made by rubbing up a little finely pulverised chalk, preferably precipitated chalk (but prepared chalk or even whiting will do) with a very small quantity of vinegar or a trace of hydrochloric acid. This forms a very convenient application to bleeding gums or shaving cuts. It is also a suitable form of styptic to apply to any surface from which actual or serous hemorrhage is taking place.

\section{(5) Simple Method of TestiNg THE EfFicacy of ANY} Physiological Styptios.

The efficacy of any physiological styptic may be conveniently tested upon such a drop of blood as may be pressed out from a prick at the base of the finger-nail. The blood is to be received upon a finger-nail which has been previously warmed by passing it through a flame or by holding it above a lamp. A minute drop of the styptic (it is preferable that the styptic should be previously warmed to blood heat) is then introduced into the drop of blood. The course of coagulation in this drop is to be compared with the courge of coagulation in a control drop of blood obtained from the nezt finger. If the styptic possesses a satisfactory degree of efficacy it will then be found that a definite clotting will be obtained in the drop of blood which has been operated upon after the lapse of a full minute. In this interval no sign of clot will be obtained in the control drop. On the contrary it will be found that in practically all cases the control drop will dry up without showing any sign of clotting. ${ }^{2}$

(6) Method of Warming on Cooling CoagulationTUBES FOR BLOOD COAGULABILITY ESTIMATIONS TO a Standard Temperature.

The method of determining the coagulability of the blood which was proposed by $\mathrm{me}^{3}$ for clinical uses consists in filling in a series of six or eight capillary coagulation-tubes with blood which is obtained from the tip of the finger. In order to secure that the results which are obtained by this method should be comparable I proposed, first, that the coagulationtubes of an internal diameter of $0.25 \mathrm{~mm}$. or $\frac{2}{10}$ in. should always be employed ${ }^{*}$; and, secondly, that the blood coagulability estimations should always be undertaken at a temperature of half blood heat $\left(185^{\circ} \mathrm{C}\right.$. or $\left.66^{\circ} \mathrm{F}.\right) .^{5}$ When the temperature is either considerably above or considerably below the suggested normal it becomes necessary to warm or cool the coagulation-tubes. It was originally proposed that this readjustment of temperature should be effected by placing the tubes in contact with the outer wall of a vessel of cold or warm water. ${ }^{6}$ Further experience has, however, shown that the readjustment of temperature may be effected in the following simpler manner.

Rabber caps are first drawn over the ends of the coagulation-tubes. The tubes are then placed upright in a tumbler of water at a temperature of $185^{\circ} \mathrm{C}$. When they have taken on the exact temperature of the water, the tubes are taken out of the tumbler, and the outside of the rubber caps is carefally dried. The caps can now be removed without any fear of water getting into the interior of the tube. The tubes are now filled in with blood from the finger. In view of the fact that thick glass is employed in making these coagulation tabes no further precautions need in an ordinary case be taken with regard to the temperature. An exception to this rule has, however, to be made, first, where the temperature of the surrounding air is either very hot or very cold, and, secondly, where-as for instance in bæmophilia-we are dealing with cases in which blood coagulability is inordinately reduced. In cases of this kind it is essential that the blood should be kept at the standard

2 The best way of detecting traces of clot in a drop of blood is to wipe off the drop with a clean bandkerchief or with a piece of tilter paper. If any coagulation has taken place shreds of fibrin will be detected on the

3 The Laxcet, Dec. 2nd, 1893, p. 1390. Brit. Med. Jour., Jul5, 1893, and February 1894.

4 These can be obtained from Mr. A. E. Dean, jun., 73, Hatton-

temperature was selected because it approximates rery closely to the ordinary temperature of dwelling rooms and hospital wards in temperate climates. 
temperature during the whole course of the observation. This may readily be done by returning the tubes to the tnmbler of water after they have been filled in with blood. This can be done without any danger of introducing water tnto the blood if, in conformity with the prescribed routine, the blood has been withdrawn some little distance up from the orifice of the tube. The intervening bubble of air will then not only prevent the mixture of any water with the blood, but it will further so increase the friction that the column of blood will not be driven further up the tube by the pressure of the surrounding water.

Netiey.

\section{PAROXYSMAL HYPER-ACIDITY IN CHILDREN SIMULATING MIGRAINE. ${ }^{1}$}

BY W. SOLTAU FENWICK, M D , M.R C.P. LOND., PHYSICLAN TO OLP-PATIEYTS AT THE EVELINA HOSPITAL FOR SIC

CHLLIREX; PHYSICIAN IO THE LOYDON TEMPERANCE HOSPIIAL.

CHRONIC disorders of the stomach accompanied by an excessive secretion of the gastric juice are rarely encountered below the age of puberty. Intermittent hyper-acidity, on the other hand, does not appear to be an uncommon phenomenon in early life. Thus Rossbach, ${ }^{2}$ Rosenthal, ${ }^{3}$ Lépine, ${ }^{4}$ and others have described cases in which severe headache and other cerebral symptoms were associated with an abnormal secretion of hydrochloric acid and seem to consider that in many instances at any rate the gastric disorder was the primary complaint. In this country cases of a similar nature are occasionally observed, but the disease appears to be much less common than on the Continent or in America, for out of some 3000 consecutive cases of disordered digestion in children which I have examined only five exhibited symptoms similar to those of migraine associated with hyper-acidity of the gastric juice. Although the disease is therefore a rare one it presents so many features of clinical interest that it seems to merit a more detailed description than is usually accorded it.

Symptoms.-The disorder may commence at any period of childhood, but is most common between the ages of four and ten. As a rule an attack is immediately preceded by a period of unusually good health, but occasionally the child suffers from malaise with want of appetite and constipation for several days before the headache commences. Mental and physical fatigue are the chief conditions which appear to induce an attack, although over-indulgence in sweets or prolonged excitement is sometimes responsible for its development. An attack usually begins with severe headache which makes its appearance either in the early morning or shortly after the midday meal. At first the pain is confined to the frontal or occipital region, but it soon becomes diffased over the entire cranium and is often accompanied by tender ness of the scalp. Any attempt to stand upright, to move the head, or even to congh, increases the pain, and the child often screams out at intervals as though he were suffering from meningitis or becomes partially aphasic. After the lapse of an hour or two the patient experiences a burning pain in the epigastrium and will flex the knees and clasp the hands over the abdomen in his efforts to obtain relief. As the pain increases in intensity the stomach and intestines become distended with gas and pyrosis attended by a scalding pain behind the sternum is experienced from time to time. Flatulent eructations, nausea, and giddiness now supervene and the child suddenly sits op and empties his stomach without apparent effort. The act of emesis is followed by pain in the throat and choking and leaves a sour and unpleasant taste in the mouth. In some cases both the headache and abdominal pain subside immediately the stomach has been emptied, so that the patient falls asleep and awakes in a few hours quite restored to health. As a rule, however, retching and vomiting continue for many hours and convalescence is

1 Abstract of a lecture delivered at the London Temperance Hospital, Nov. 25th, 1897

2"Gastroxynsis," Deutsches Archiv für Klinische Medicin, 1884, 3. 383. Tomitus Hyperacidus," Berliner Klinische Wochenschrift, 1887, 4 "Gastroxie," Société Médicale des Hôpitaux, 1885, p. 134. See also Snow, Archiv für Pediatrie, 1893, p. 986. not established for two or three days. During the painful crisis the face and extremities are cold and the patient often shivers or complains of numbness and tingling in the fingers or feet. The pulse is small and often abnormally slow, especially at the commencement of the attack. As a rule the temperature of the body is depressed, but occasionally the thermometer in the rectum may register $100^{\circ}$ or $101^{\circ} \mathrm{F}$. for a fe $x$ hours. In most cases the bowels are confined, but sometimes the initial vomiting is replaced by a sharp attack of diarrhoa, which appears to exert the same beneficial influence upon the cerebral symptoms. As long as the disorder persists the appetite rtmains in abeyarce, but thirst is often a marked symptom of the case. In the early stages the tongue is clean and moist, but if the vomiting proves severe the organ soon becomes coated with a creamy far. It is an interesting fact that at the commencement of an attack the administration of a tumblerful of tepid water often relieves both the headache and epigastric pain and in many cases this simple procedure is sufficient to effect an immediate cure. At an Jater stage, however, the ingestion of water merely excites vomiting. The vomit varies in character according to the time of day at which the emesis commences. If the attack originates in the early morning when the stomach is devoid of food the ejecta consist of a greenish-yellow fluid mixed with mucus; but should the vomiting occur after the midday meal particles of food are abondant and exhibit signs of partial digestion. In each case the total acidity of the ejecta is unduly high from the presence of an excess of free bydrochloric acid. If the attack follows the mid-day meal the vomit often possesses an acidity equal to from 045 to 0.52 per cent. bydrochloric acid; but when it ensues in the early morning the total acidity of the gastric contents may not exceed 038 per cent. hydrochlolic acid. If the vomiting continues after the stomach has been emptied the ejecta gradually lose their acidity until at length they consist entirely of alkaline and bile-stained mucus. After the disorder has subsided the secretion of bydrochloric acid is found to be normal.

Diagnosis.-Unless the romit is submitted to a chemical examination the aisease may easily be confonnded with migraine or recurrent catarrh of the stomach. It may be observed, however, that migraine usually occurs at a later period of life than paroxysmal byper-acidity and that its onset is preceded by ocular phenomena which are absent in the latter disorder. The headache also is never relieved by drinking tepid water nor is severe epigastric pain an ordinary symptom of the complaint. Lastly, the ejecta never contain any excess of free hydrochloric acid. Gastric catarrh is an extremely common complaint in infancy and childhood and closely resembles paroxysmal hyper-acidity in many of its symptoms. The headache, however, is much less violent, epigastric pain is either slight or absent altogether, while nausea and retching constitate the chief features of the complaint. The vomit consists entirely of bile-stained mucus and never exhibits any trace of free byìrochloric acid.

Treatment.-All conditions which tend to excite an attack must be avoided as far as possible and in severe cases the child should be kept from school and be encouraged to indulge in plenty of exercise in the fresh air. The meals should be taken at regular intervals and all fatty and saccharine articles be excluded from the dietary. If the bowels are confined they should be regulated by means of some simple laxative, such as cascara, senua, sulphur and guaiacum, or compound liquorice powder. At the commencement of an attack the child should be put to bed and the room be darkened. In many cases an emetic of ipecacuanha wine or sulphate of zinc relieves the headache more quickly than anything else. Occasionally a full dose of antipyrin or phenacetin will cut short an attack if administered at its commencement. If vomiting has already taken place lavage of the stomach can be promoted by making the patient drink warm water containing a small quantity of bicarbonate of sodium and afterwards exciting vomiting by tickling the back of the throat. The employment of the stomach-tube for this purpose is never required. In those cases where catarrh of the stomach ensues from the irritant effects of the acid secretion and the child continues to retch for many hours an enema of chloral and bromide of potassium may be administered. Among the varions remedies which have been tried for the purpose of preventing a recurrence of the disease bromide of potassium combined with Jiquor potassæ has proved of the greatest value.

Devonshire-street, $W$. 